\title{
XFEL EXPERIMENTS: JITTER OF PUMP-PROBE TIME DELAYS AND PULSE INTENSITIES
}

\author{
S. Bratos ${ }^{1}, \mathrm{M}$. Wulff ${ }^{2}$, and J-Cl. Leicknam ${ }^{1}$ \\ 1 - Sorbonne Universités, UPMC Univ Paris 06, Laboratoire Physique Théorique de la \\ Matière Condensée, 75005, Paris, France. \\ 2 - ESRF - European Synchrotron, Complex Systems and Biomedical Sciences (CBS) CS \\ 40220, 38043 Grenoble Cédex 9, France.
}

\section{SUMMARY}

Jitter of XFEL signals due to fluctuations in shot-to-shot time delays and intensities are explored in the frame of a statistical theory of X-ray diffraction from liquids. Deformed signals are calculated at different levels of pump-probe jitter. A new method is proposed to eliminate these distortions.

I. INTRODUCTION. Monitoring atomic motions during a chemical reaction has always been an important objective in chemical research. This sort of "filming", inaccessible in the past, can now be realized either by performing time-resolved optical or time-resolved x-ray experiments. Optical experiments, less expensive than $\mathrm{x}$-ray experiments, were realized first and they proved to be highly efficient. The Nobel prize for chemistry was awarded to A. Zewail for his spectacular achievements in this field [1]. However, as the wave length of optical waves are large compared with inter-atomic distances in molecules, optical techniques can not detect atomic positions without complementary assumptions. This difficulty is absent in X-ray experiments. They can be realized, both in diffraction or absorption, either using synchrotron or free electron laser (XFEL) techniques. Pulses of the order of 100 ps can be generated by the former, and 10 fs by the latter. X-ray techniques, in particularly XFEL techniques have proven to be extremely efficient, but a number of difficulties still limit, for the time being, their intrinsic power: the shot-to shot dispersion of pump-probe time delays and of pulse intensities. An important efforts has been made to solve this problem experimentally [2][3][4][5][6][7][8][9]. The recent measure and sort technique [10] merits attention in this context. We complete this effort theoretically by calculating the signal distortions in some typical situations. We also propose a new method to eliminate these distortions.. 
37 II. THEORY. (a) In a time-resolved X-ray experiment, the sample is pumped by 38 an optical pulse and probed by an X-ray pulse. The pump-probe time delay must be determined with extreme accuracy. At the present time, while XFEL sources

40 generate pulses down to $10 \mathrm{fs}$, there is a jitter on the pump-probe time delays of

41 several hundreds fs. The experiment must thus be repeated and the resulting

42 signals averaged over this sequence to make the results usable. In this way, a

43 single-pulse experiment transforms into an multi-pulse experiment. The problem 44 is thus statistical, not only in its molecular dynamics part, but also in the electric 45 field part. Statistical mechanics is thus omnipresent, as in ultrafast optical

46 spectroscopy; see e.g. the text book by Mukamel [11].

47 (b) A statistical theory of $\mathrm{x}$-ray diffraction from liquids was published some time 48 ago [12]. Its full mathematical development is given in this reference, and will 49 not be repeated again. Only the essential features are illustrated in what follows. 50 The intensity of the diffracted $\mathrm{x}$-rays $\Delta \mathrm{S}(\mathrm{q}, \tau)$ is:

$$
\begin{gathered}
\Delta S(\boldsymbol{q}, \tau)=\int_{-\infty}^{\infty} d t I_{X}(t-\tau) \Delta S_{\text {inst }}(\boldsymbol{q}, t) \\
\Delta S_{\text {inst }}(\boldsymbol{q}, t)=\left(\frac{e^{2}}{m c^{2} \hbar}\right)^{2} P \int_{0}^{\infty} \int_{0}^{\infty} d \tau_{1} d \tau_{2}\left\langle E_{i}\left(\boldsymbol{r}, t-\tau_{1}\right) E_{j}\left(\boldsymbol{r}, t-\tau_{1}-\tau_{2}\right)\right\rangle_{O} \\
\boldsymbol{x}\left\langle\left[\left[f_{m} f_{n} \mathrm{e}^{-i \boldsymbol{q} \cdot \boldsymbol{r}_{m n}\left(\tau_{1}+\tau_{2}\right)}, M_{i}\left(\tau_{2}\right)\right], M_{j}(0)\right]\right\rangle_{S}
\end{gathered}
$$

51 Here $\mathrm{P}$ is a factor characteristic of the experimental set-up such as the temporal 52 pulse profile, polarisation, sample concentration, etc. $\mathrm{I}_{\mathrm{X}}$ is the intensity of the

53 incident $X$-ray radiation, $E_{i}, E_{j}$ are components of the electric field generated by

54 the optical laser, $\mathbf{q}$ is the wave vector, $\mathrm{f}_{\mathrm{m}}, \mathrm{f}_{\mathrm{n}}$ are atomic scattering factors, $\mathrm{r}_{\mathrm{m}}$ is

55 the distance between the atoms $m$ and $n$, and $M_{i}, M_{j}$ are components of the laser

56 induced transition moment $M$ between the states $\mathrm{i}$ and $\mathrm{j}$. Einstein's convention

57 of summing over doubled indices $i, j$ and $m, n$ is employed. The form of this

58 expression can be understood comparing it with the standard expression for the

59 diffracted $\mathrm{x}$-ray intensity $\mathrm{S}(\mathrm{q}) \sim \Sigma_{\mathrm{m}, \mathrm{n}}\left[\mathrm{f}_{\mathrm{m}} \cdot \mathrm{f}_{\mathrm{n} .} \exp \left(-\mathrm{iq} \cdot \mathrm{r}_{\mathrm{mn}}\right)\right]$ [13]. The later is valid if

60 the incident $X$ ray wave has a constant amplitude and if fast chemical processes

61 are absent. If the incident X-ray consists of short pulses, and if some fast

62 chemical process is laser excited, this expression must be modified in two ways.

63 First, the intensity and the inter-atomic distances $r_{m n}$ are now time dependent,

64 and $I_{X}$ and $r_{m n}$ must be replaced by $I_{x}(t)$ and $r_{m n}(t)$.The remaining quantities in

65 Eq.(1) describe the laser induced electronic excitation. This can be understood

66 noticing that, according to the Fermi golden rule, the rate of this excitation is

67 proportional to $1 / \hbar^{2}(\mathbf{E} . \mathbf{M})^{2}$, where $\mathbf{E}$ is the laser generated electric field and $\mathbf{M}$ 
68 the transition moment. The presence in Eq. (1) of the factors $1 / \hbar^{2}, \mathrm{E}\left(\mathrm{t}-\mathrm{\tau}_{1}\right), \mathrm{E}\left(\mathrm{t}-\mathrm{t}_{1}\right.$ $\left.69 \tau_{2}\right), M(0)$ and $M\left(\tau_{2}\right)$ can be understood in this way. The connection of different

70 time points can not be explained as simply. This equation can be used as it

71 stands when studying single pulse events.

72 Interpreting multi-pulse experiments is more complex, due to the scatter of 73 pump-probe time delays and shot intensities. However, the form of Eq. (1)

74 indicates that these problems can be studied independently from those due to

75 molecular dynamics. Note also that Eq. (1) was conceived for a single pulse

76 experiment. However, a slight modification makes it applicable to a multi-pulse

77 experiment: it is sufficient to replace the single $X$-ray pulse intensity $I_{X}(t-\tau)$ by

78 the average multi-pulse intensity $\left.<\mathrm{I}_{\mathrm{X}}(\mathrm{t}-\mathrm{\tau})\right\rangle_{\mathrm{MP}}$, the index MP indicating multi-

79 pulse. One can then write:

$$
\Delta S(q, \tau)=\int_{-\infty}^{\infty} d t\left\langle I_{X}(t-\tau)\right\rangle_{M P} \Delta S_{i n s t}(q, t)
$$

80 where $\Delta S_{\text {inst }}(q, t)$ is the same as in Eq. (1). In the rest of this paper, the incident

81 x-ray beam is supposed to be Gaussian:

$$
I_{X}(t-\tau-\delta \tau)=I \exp \left[-\gamma_{X}(t-\tau-\delta \tau)^{2}\right]
$$

82 where $\tau$ is the nominal pump-probe time delay, $\delta \tau$ its ill controlled shut-to-shut

83 time increment and $\left(1 / \gamma_{\mathrm{x}}\right)^{1 / 2}$ its temporal width.

84 c) To proceed further, details about the statistical distribution of $\delta \tau$ and I for 85 subsequent shots are required. The attention of the experimentalists was centered 86 on this question for years, and still remains an issue. According to the literature

87 [14], the distribution of pump-probe time delays $\mathrm{P}(\delta \tau)$ is Gaussian:

$88 P(\delta \tau)=\sqrt{(\beta / \pi)} \exp \left(-\beta(\delta \tau)^{2}\right)$. The distribution of shot-to shot intensitires $\mathrm{P}(\mathrm{I})$ is

89 less well known, but according to Eqn(2) it is needed only if the absolute

90 intensity of the scattered radiation is explored, which is not the case here. Then,

91 inserting Eqn(3) into Eqn(2) and integrating over $\delta \tau$, there results:

$$
\Delta S(q, \tau)=I\left(\frac{\beta}{\beta+\gamma_{X}}\right)^{\frac{1}{2}} \int_{0}^{\infty} d t \exp \left(-\left(\frac{\beta \gamma_{X}}{\beta+\gamma_{X}}\right)(t-\tau)^{2}\right) \Delta S_{\text {inst }}(q, t)
$$


92 Jitter thus generates an effective temporal broadening of incident x-ray pulses.

93 This is the basic equation relating the distorted and non-distorted signals

$94 \Delta S(q, \tau)$ and $\Delta S_{\text {inst }}(q, t)$, respectively.

95 To proceed further, the following way can be chosen. Let the laser excitation

96 promote the molecules from their ground electronic state 0 , where the length of

97 a given bond is $r_{0}$, to an electronic state 1 , where it is $r_{1}$. According to the

98 Franck-Condon principle, $r_{1}(0)=r_{0}$ at time $\mathrm{t}=0$. The simplest assumption

99 to describe the bond length variation at later times consists in writing

$100 r(t)=r_{1}-\left(r_{1}-r_{0}\right) \cdot \exp \left(-t / \tau_{r}\right)$ where $\tau_{r}$ is the molecular reaction (or

101 rearrangement) time (Fig.1). The signal $\Delta S_{\text {inst }}(q, t)$, non affected by pump-

102 probe time delay dispersion, can be written:

$$
\Delta S_{\text {inst }}(q, t)=\frac{\sin \left(q\left(r_{1}-\left(r_{1}-r_{0}\right) \exp \left(-t / \tau_{r}\right)\right)\right)}{q\left(r_{1}-\left(r_{1}-r_{0}\right) \exp \left(-t / \tau_{r}\right)\right)}-\frac{\sin \left(q r_{0}\right)}{q r_{0}}
$$

103 Then, inserting Eqn(5) into Eqn(4) and integrating provides $\Delta S(q, \tau)$.The 104 integration can be performed either numerically or analytically if $r_{1}-r_{0} \ll r_{0}$.

105 Note that this condition is not very restrictive. When passing from a single C-C 106 bond to a triple $\mathrm{C}-\mathrm{C}$ bond, $\mathrm{r}_{0}=1.5 \mathrm{~A}$ and $\mathrm{r}_{0}-\mathrm{r}_{1}=0.3 \mathrm{~A}$. The experimental signal $107 \Delta S(q, \tau)$ can then be calculated and its distortion investigated, if the 108 parameters $r_{1}$ and $\tau_{r}$ are known. The opposite problem of extracting the 109 non perturbed signal $\Delta S_{\text {inst }}(q, t)$ from the observed signal $\Delta S(q, \tau)$ is more 110 difficult. The best is to work with the function $\Delta S(q, \tau)$ in its analyitcal form:

$$
\begin{gathered}
\Delta S(q, \tau) \sim-q\left(r_{1}-r_{0}\right) \cdot j_{1}\left(q r_{0}\right) \\
\cdot\left[\operatorname{erfc}\left(-\sqrt{\frac{\beta \cdot \gamma_{X}}{\beta+\gamma_{X}}} \tau\right)\right. \\
-\operatorname{erfc}\left[\left(\frac{1}{2 \tau_{r}}\right) \sqrt{\frac{\left(\beta+\gamma_{x}\right)}{\beta \gamma_{x}}}-\sqrt{\left.\left.\frac{\beta \cdot \gamma_{X}}{\beta+\gamma_{X}} \tau\right] \exp \left(\left(\frac{1}{4 \tau_{r}^{2}}\right) \frac{\beta+\gamma_{x}}{\beta \gamma_{x}}-\frac{\tau}{\tau_{r}}\right)\right]}\right.
\end{gathered}
$$

111 where $r_{0}$ and $r_{1}$ are bond lengths before and after reaction, $\tau_{r}$ is its characteristic

112 time and $\mathrm{j}_{1}(\mathrm{x})$ is the Bessel function of the order 1 ( remember that $\sin \mathrm{x} / \mathrm{x}$ is the

113 Bessel function $\mathrm{j}_{0}(\mathrm{x})$ ). Inserting experimental data into the left hand member of

114 Eqn(6) then permits to calculate $r_{1}$ and $\tau_{r}$ using mean square optimisation

115 techniques. As there are only two prameters $r_{1}$ and $\tau_{r}$ to determine, this

116 calculation is easy. 
117 The corresponding $r$ space signals $\Delta S[r, \tau]$ can be calculated by Fourier 118 inverting $\Delta S(q, \tau)$. This can be done without any special precaution if $\tau$ is 119 large as compared with the time $\tau<1 / \sqrt{\beta}$ characteristic of pump-probe 120 dispersion. If this is not the case, $\Delta S(q, \tau)$ must be corrected carrying out the 121 above procedure for each q, $\tau$ point such that $\tau<1 / \sqrt{\beta}$, this making the Fourier

122 transform possible. It is thus more difficult to correct the signals $\Delta S[r, \tau]$ than 123 the signals $\Delta S(q, \tau)$.

124 III. EXAMPLES (a) Times shorter than the molecular dynamics. Those 125 considered here are of the order of $10 \mathrm{fs}$ or shorter. At these times a liquid 126 behaves like a glass. Nevertheless, diffraction signals still vary with time, even if 127 all inter-atomic distances $r$ are fixed. This is due to the electric fields $E_{i}, E_{j}$ of the 128 optical pump pulses in Eq. (1). The noise of XFEL radiation also plays a major 129 role. In this limit, one finds :

$$
\Delta S_{X F E L}(\tau)=\text { Const.erfC }\left(-\sqrt{\left(\frac{\beta \gamma_{X}}{\beta+\gamma_{X}}\right)} \tau\right)
$$

131 One concludes that the dispersion of pump probe time delays modifies the 132 temporal width of the average multi-pulse signals even at very short times.

133 These effects may be large, even overwhelmingly large; compare with Fig. 2.

134 Note also that in this short-time limit the q- and r-resolved signals exhibit the

135 same tau dependence. In fact, in this limit $\Delta S_{\text {inst }}(q, t)$ is independent of time. A 136 look on Eqn(6) then confirms the statement.

137 (b) Contracting chemical bond. In absence of distortion free experimental data in 138 the 10 - 100 fs time domain, the following example is completely theoretical. 139 Let us start considering a CC bond contracting from 1.5A to 1.2A; these values 140 correspond to a single and triple CC bond respectively. This CC bond is 141 supposed to be a part of a polyatomic molecule PolyM. Its CC diffraction peak 142 is assumed to be sufficiently isolated from other diffraction peaks from PolyM

143 to be explorable. The laser pump promotes PolyM from its electronic ground 144 state A, where the CC bond is simple to a state B where it is triple. However, 145 this transformation is not instantaneous: according to the Franck-Condon 146 principle, light induced transitions are all vertical. At $\tau=0$, the CC distance 147 remains unchanged, equal to $1.5 \mathrm{~A}$. It is only at later times that it contracts 148 gradually from $1.5 \mathrm{~A}$ to $1.2 \mathrm{~A}$. How does this contraction process manifests itself 149 in a r resolved XFEL experiment? And how does this signal deform if the pump150 probe times are dispersed? The central quantities are the pair distribution 
151 functions $g(r, t)$; see the textbook [Hansen,1997]. The following expressions are 152 chosen in our model:

$$
\begin{gathered}
\mathrm{g}_{\mathrm{B}}(\mathrm{r}, \mathrm{t})=\begin{array}{l}
\mathrm{g}_{\mathrm{A}}=\sqrt{a_{A} / \pi} \exp \left[-\mathrm{a}_{\mathrm{A}}\left(\mathrm{r}-\mathrm{r}_{\mathrm{A}}\right)^{2}\right] \\
\sqrt{a_{B} / \pi} \exp \left[-\mathrm{a}_{\mathrm{B}}\left(\mathrm{r}-\mathrm{r}_{\mathrm{B}}-\delta \mathrm{r}_{\mathrm{B}} \exp \left(-\mathrm{t} / \mathrm{\tau}_{\mathrm{v}}\right)\right)^{2}\right] \\
\mathrm{n}_{\mathrm{A}}(\mathrm{t})=1-\mathrm{n}_{0} \cdot \exp \left(-\mathrm{t} / \mathrm{\tau}_{\mathrm{p}}\right) \\
\mathrm{n}_{\mathrm{B}}(\mathrm{t})=\mathrm{n}_{0} \cdot \exp \left(-\mathrm{t} / \mathrm{\tau}_{\mathrm{p}}\right)
\end{array}
\end{gathered}
$$

153 Note that $g_{A}(r)$ and $g_{B}(r, t)$ approach a delta function when $a_{A}$ et $a_{B}$ go to infinity.

154 Equation (7b) states that the CC bond contracts in the state B of PolyM in times

155 of the order of $\tau_{v}$. Employing the above equations together with Eqs. (2, 4)

156 generates the r-resolved signal $\Delta \mathrm{S}(\mathrm{r}, \mathrm{\tau})$.

157 The parameters of the above model are: the ground state distance $\mathrm{r}_{\mathrm{A}}$ is $1.5 \mathrm{~A}$ and

158 the excited states distance $\mathrm{r}_{\mathrm{B}} 1.2 \mathrm{~A}$; the laser induced contraction of the CC bond

159 in the state $\mathrm{B}$ of PolyM is $0.3 \mathrm{~A}$. The parameters $\mathrm{a}_{\mathrm{A}}$ and $\mathrm{a}_{\mathrm{B}}$ are both of the order

160 of $25 \mathrm{~A}^{-2}$, which corresponds to a half width of $g_{A}(r)$ and $g_{B}(r, t)$ of the order of

$1610.4 \mathrm{~A}$. Moreover, the recombination time $\tau_{\mathrm{v}}$ is assumed to be of the order of 100

$162 \mathrm{fs}$, and the population relaxation time $\tau_{p}>>\tau$. These values correspond to an

163 ultrafast chemical process.

164 The results are presented now. Fig. 3a illustrates $\Delta S[r, \tau]$, the r-resolved XFEL

165 signal of a contracting CC bond in absence of pump-probe time dispersion. This 166 signal is presented in three dimensions: the distance $r$ and the time $\tau$ are defined 167 on the two coordinate axes while the intensity is given by color. The red valley 168 at 1.5A pictures the deficit of CC bonds at the initial bond length of $1.5 \mathrm{~A}$, 169 whereas the violet ridge indicates CC bonds of given length $\mathrm{r}$ at a given time $\tau$.

170 Note that the intensity of the differential signal is vanishing at $\tau=0$ : according

171 to the Frank-Condon principle electronic transitions are vertical. At times

172 tau 10 fs, intramolecular dynamics of PolyM intervene noticeably. It is only at

173 times tau $>20$ fs that chemistry manifests itself predominantly. The signal

174 represents a film of a contracting CC bond. If pump-probe times are dispersed,

175 the above picture is slightly or deeply modified; see Fig. $3 \mathrm{~b} \Delta \mathrm{S}[\mathrm{r}, \mathrm{\tau}]$ is only

176 blurred. It is only blurred if the pump-probe time dispersion is small. If the

177 pump-probe time dispersion is not sufficiently small, the CC contraction is no

178 longer observable and only an instantaneous jump between the initial and final 
179 configurations is observed . This effect is widely known in other fields of 180 physics and chemistry under the name of motional narrowing.

181 So much for r-resolved signals $\Delta S[r, \tau]$. Let us now pass to the q-resolved 182 signals $\Delta S(q, \tau)$. The latter can be deduced from $\Delta S[r, \tau]$ using the well known 183 formula $\Delta S(q, t)=4 \pi / q \int_{-\infty}^{+\infty} d r r \Delta S[r, t] \sin (q r) \quad$ which, according to the basic

184 theory of $\mathrm{x}$-ray diffraction, relates r-resolved and q-resolved signals

185 [Warren,2005]. It is valid independently of whether pump-probe time delays

186 are dispersed or not. This integration was accomplished numerically. The results

187 are presented in Figs. 4. In Fig. 4a, the signal is calculated for $\beta=$ infinite, i.e. in

188 absence of pump-probe dispersion . It is presented in three dimensions: the

189 variables $q$ and $\tau$ are placed on the coordinate axes, whereas the value of the

190 signal $\Delta S(q, \tau)$ is indicated by color. The violet crests indicate the increase of

191 the signal intensity and the red valleys their decrease. The bending of the red

192 crests toward large q's indicates progressive CC contraction from 1.5A to 1.2

193 A. This signal is strictly vanishing at $\tau=0$, whatever $q$, which is a

194 consequence of the Franck-Condon principle. At long times, $\Delta \mathrm{S}(\mathrm{q}, \tau)$

195 approaches the limit Const $\left(r_{1}^{2} \sin \left(q r_{1}\right) / q r_{1}-r_{0}^{2} \sin \left(q r_{0}\right) / q r_{0}\right)$ (Fig.4b). Atomic

196 motions during a chemical reaction can thus be monitored in this way.

197 Nevertheless, visualizing atomic motions is much more difficult in q-resolved

198 than in r-resolved signals. Fig. 4b pictures this signal in presence of appreciable

199 pump-probe time dispersion. Only immutable red and violet crest are now

200 visible, molecular dynamics can no longer be followed. Motional narrowing is

201 dominating.

202 IV. CONCLUSION. Fluctuations of a multi-pulse signal due to of shot-to-shot

203 variations in time delays and intensities are explored theoretically in the frame

204 of a statistical theory of X-ray diffraction of liquids. A new method is also

205 proposed to eliminate the effect of time delay jitter in XFEL experiments.

206 Contrary to the measure and sort method which is fully experimental, the

207 present method belongs to the ensemble of signal treatment methods. It does not 208 require any extra experiment. 
1: A. H. Zewail, Femtochemistry. Past, present, and future, Pure and Applied Chemistry, (2000) 2219-2231.

2: M. Meyer, D. Cubaynes, P. O'Keeffe, H. Luna, P. Yeates, E. T. Kennedy, J.

T.Costello, P. Orr, R. Taïeb, A. Maquet, S. Düsterer, P. Radcliffe, H. Redlin, A.Azima, WB. Li5, E. Plönjes, and J. Feldhaus, Twocolor photoionization in xuv freeelectron andvisible laser fields, Phys. Rev. A , (2006) 011401.

3: D. M. Fritz, D. A. Reis, B. Adams, R. A. Akre, J. Arthur, C. Blome, P. H.

Bucksbaum, A. L. Cavalieri, S. Engemann, S. Fahy, R. W. Falcone, P. H. Fuoss, K. J. Gaffney, M. J. George, J. Hajdu, M. P. Hertlein, P. B. Hillyard, M. Horn-von Hoegen, M. Kammler, J. Kaspar, R. Kienberger, P. Krejcik, S. H. Lee, A. M. Lindenberg, B. McFarland, D. Meyer, T. Montagne, É. D. Murray, A. J. Nelson, M. Nicoul. Pahl, J. Rudati, H. Schlarb, D. P. Siddons, K. Sokolowski-Tinten, Th. Tschentscher, D. von der Linde, J. B. Hastings, Ultrafast Bond Softening in Bismuth: Mapping a Solid's Interatomic Potential with X-rays, Science, (2007) 633-636.

4: T. Maltezopoulos, S. Cunovic, M. Wieland, M. Beye, A. Azima, H. Redlin, M. Krikunova, R. Kalms, U. Frühling, F. Budzyn, Single-shot timing measurement of extreme-ultraviolet free-electron laser pulses, New Journal of Physics, (2008) 033026.

5: A. Azima, S. Düsterer, P. Radcliffe, H. Redlin, N. Stojanovic, W. Li, H. Schlarb, J. Feldhaus, D. Cubaynes, M. Meyer, J. Dardis, P. Hayden, P. Hough, V Richardson, E. T. Kennedy, and J. T. Costello, Time-resolved pump-probe experiments beyond the jitter limitations at FLASH, Applied Physics Letters, (2009) 144102.

6: J. M. Glownia, J. Cryan, J. Andreasson, A. Belkacem, N. Berrah, C. I. Blaga, C. Bostedt, J. Bozek, L. F. DiMauro, L. Fang, J. Frisch, O. Gessner, M. Gühr, J. Hajdu, M. P. Hertlein, M. Hoener, G. Huang, O. Kornilov, J. P. Marangos, A. M. March, B. K. McFarland, H. Merdji, V. S. Petrovic, C. Raman, D. Ray, D. A. Reis, M. Trigo, J. L. White, W. White, R. Wilcox, L. Young, R. N. Coffee, and P. H. Bucksbaum, Timeresolved pump-probe experiments at the LCLS, Optics Express, (2010) 1762017630.

7: F. Löhl, V. Arsov, M. Felber, K. Hacker, W. Jalmuzna, B. Lorbeer, F. Ludwig, K.-H. Matthiesen, H. Schlarb, B. Schmidt, P. Schmüser, S. Schulz, J. Szewinski, A. Winter, and J. Zemella, Electron Bunch Timing with Femtosecond Precision in a Superconducting Free-Electron Laser, Phys. Rev. Lett., (2010) 144801.

8: Bionta, Mina R.; Lemke, H. T.; Cryan, J. P.; Glownia, J. M.; Bostedt, C.; Cammarata, M.; Castagna, J.-C.; Ding, Y.; Fritz, D. M.; Fry, A. R.; Krzywinski, J.; Messerschmidt, M.; Schorb, S.; Swiggers, M. L.; Coffee, R. N., Spectral encoding of X-ray/optical relative delay, Optics Express 10, (2011) 21855-21865.

9: F. Tavella, N. Stojanovic, G. Geloni \& M. Gensch, Few-femtosecond timing at fourth-generation X-ray light sources, Nature Photonics , (2011) 162-165. 
10: M. Harmand, R. Coffee, M. R. Bionta, M. Chollet, D. French, D. Zhu, D. M. Fritz, H.T. Lemke,N. Medvedev, B. Ziaja, S. Toleikis, M. Cammarata, Achieving fewfemtosecond time-sorting at hard X-ray free-electron lasers, Nature Photonics, (2013) 215-218.

11: S. Mukamel, Principles of Nonlinear Optics and Spectroscopy, 1995

12: S. Bratos, F. Mirloup, R. Vuilleumier and M. Wulff, Time-resolved x-ray diffraction: Statistical theory and its application to the photo-physics of molecular iodine, J. Chem. Phys., (2002) 10615.

13: B.E. Warren, X-Ray Diffraction, 1990

14: Cavalieri, A. L., Fritz, D. M., Lee, S. H., Bucksbaum, P. H., Reis, D. A., Rudati, J., Mills, D. M., Fuoss, P. H., Stephenson, G. B., Kao, C. C., Siddons, D. P., Lowney, D. P., MacPhee, A. G., Weinstein, D., Falcone, R. W., Pahl, R., Als-Nielsen, J., Blome, C., DI"usterer, S., Ischebeck, R., Schlarb, H., SchulteSchrepping, H., Tschentscher, Th., Schneider, J., Hignette, O., Sette, F., Sokolowski-Tinten, K., Chapman, H. N., Lee, R. W., Hansen, T. N., Synnergren, O., Larsson, J., Techert, S., Sheppard, J., Wark, J. S., Bergh, M., Caleman, C., Huldt, G., van der Spoel, D., Timneanu, N., Hajdu, J., Akre, R. A., Bong, E., Emma, P., Krejcik, P., Arthur, J., Brennan, S., Gaffney, K. J., Lindenberg, A. M., Luening, K., Hastings, J. B., Clocking Femtosecond X Rays, Phys. Rev. Lett. , (2005) 114801.

15: P.A. Janson, Deconvolution of Images and Spectra, 1997

16: J.P. Hansen and I.R.M Mac Donald , Theory of Simple Liquids, 2006 
10 Bratos-Wulff-Leicknam, 9 Janvier, 2018

214

215

216

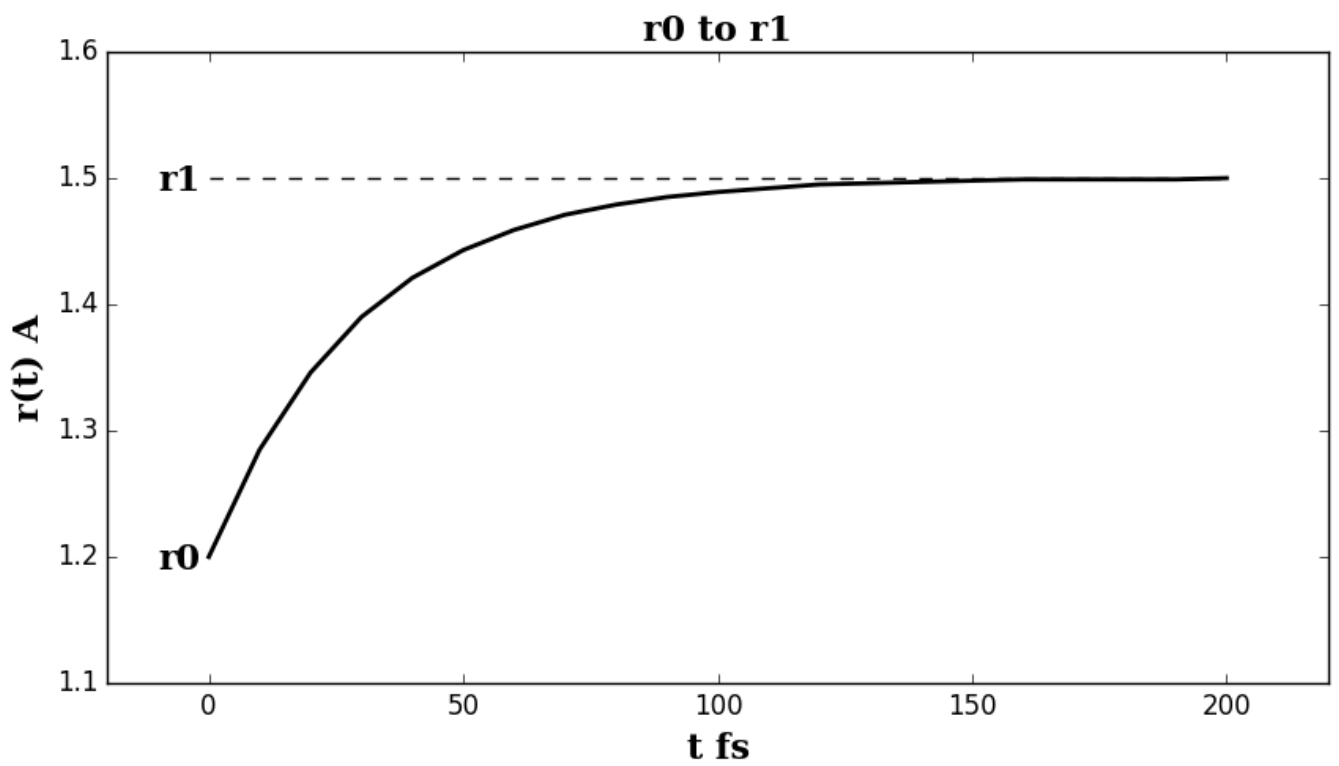

218 Fig. 1: Variation of the bond length $\mathrm{r}(\mathrm{t})$ from $\mathrm{r} 0$ to the laser excited state $\mathrm{r} 1$. 
11 Bratos-Wulff-Leicknam, 9 Janvier, 2018

219

220

221

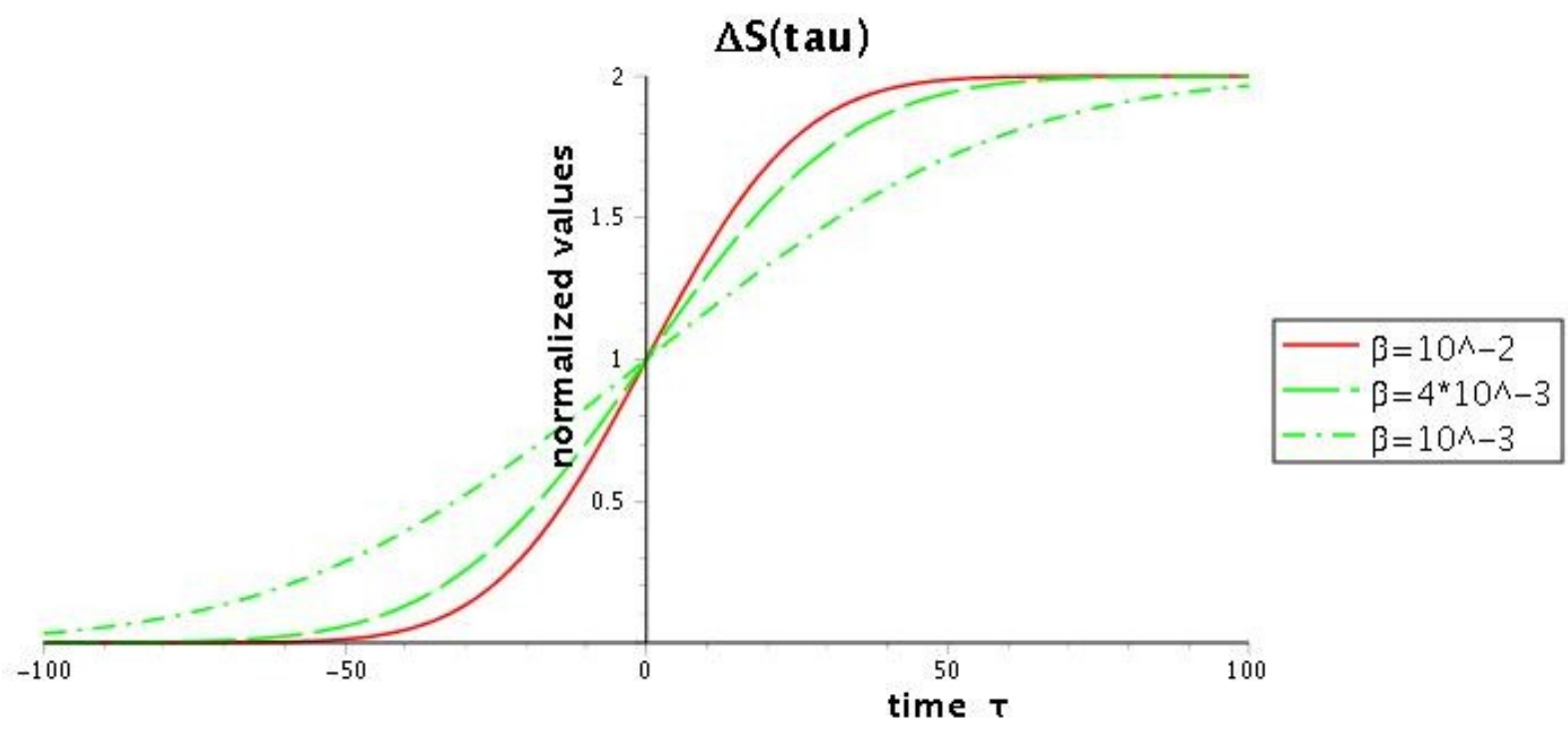

223 Fig. 2: Variation of $\Delta S$ at shortest pump-probe times delays.

224

225

226

227

228

229

230

231 
12 Bratos-Wulff-Leicknam, 9 Janvier, 2018
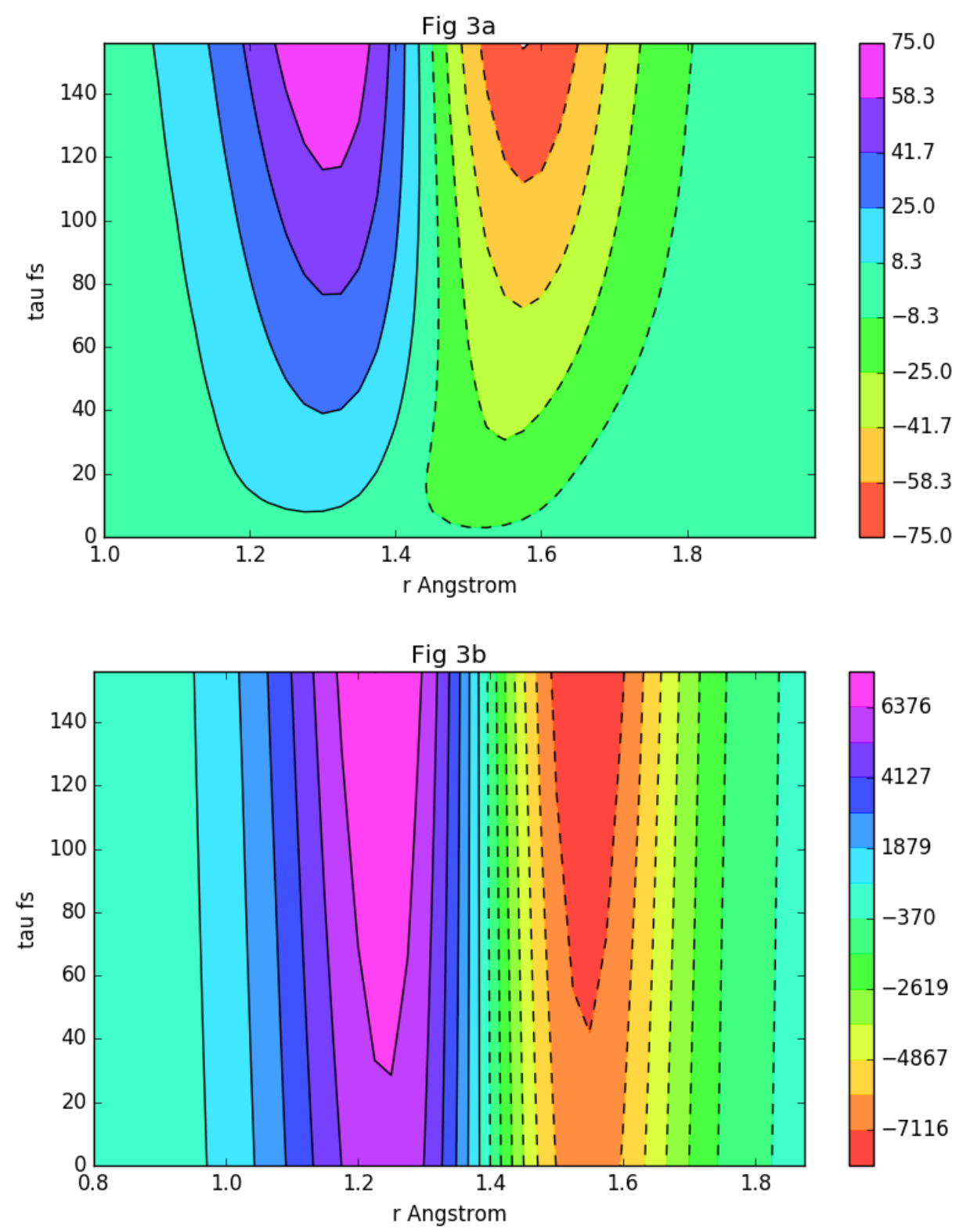

234 Fig. 3: Contraction of the CC bond after laser excitation: multi-pulse signal in $\mathrm{r}$ 235 space. The CC bond contracts from the single bond length (1.5 A) to the triple 236 bond length (1.2 A). The process is supposed to be accomplished in $100 \mathrm{fs}$. (a) 237 The signal $\Delta \mathrm{S}(\mathrm{r}, \mathrm{\tau})$ in absence of pump-probe time delay dispersion, (b) in its 238 presence (1000 fs). The contraction of the CC bond is clearly visible in Fig. (a), 239 but is not in Fig. (b). 
Fig $4 a$

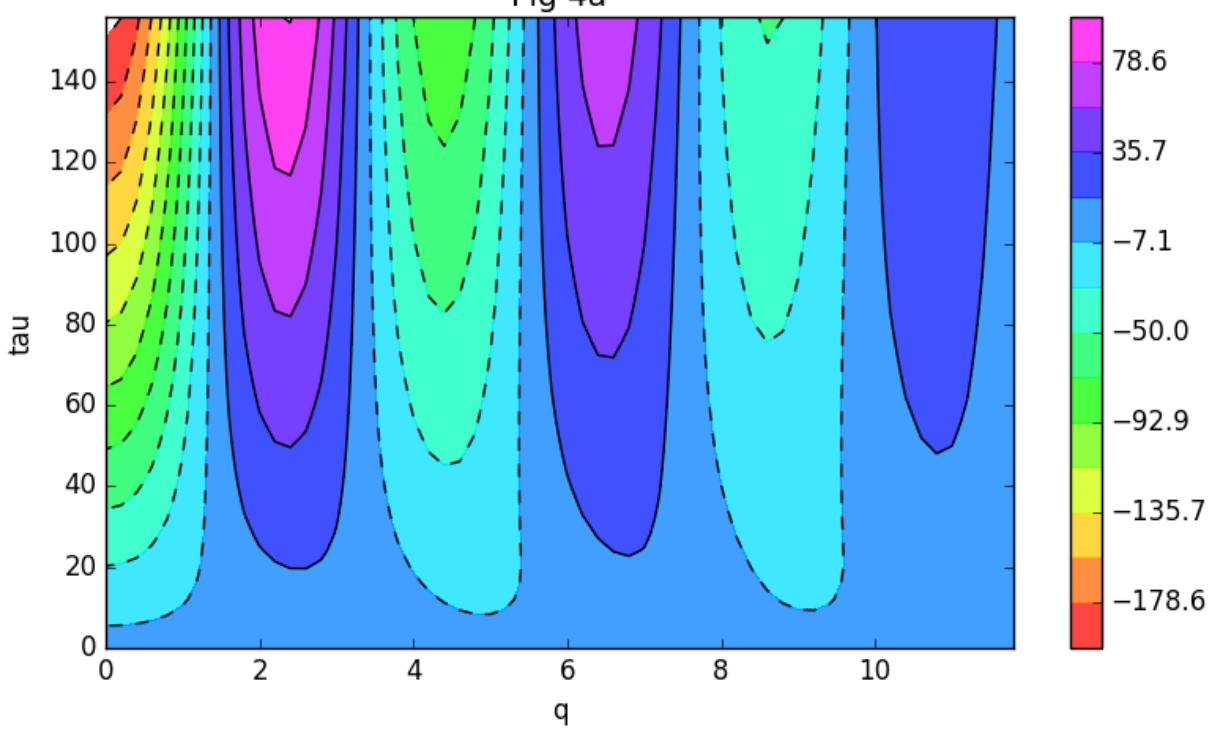

Fig $4 b$

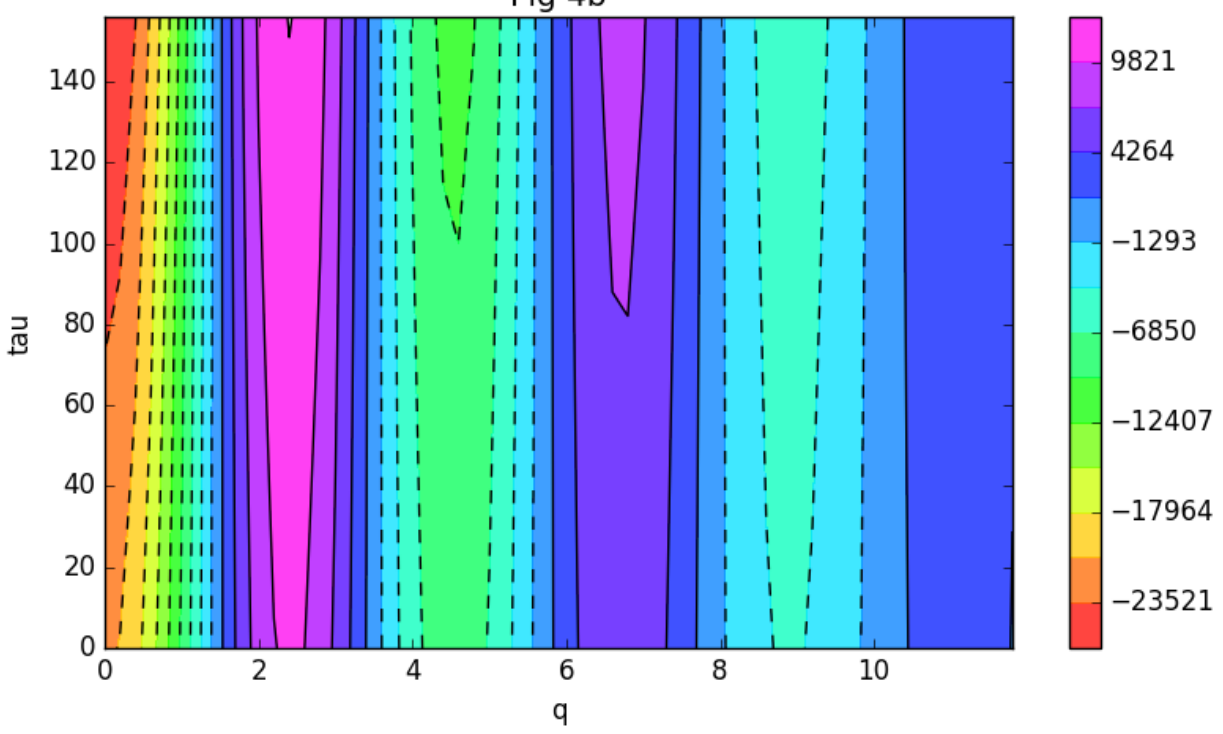

242 Fig. 4: Contraction of a CC bond after laser excitation: the multi-pulse signal in 243 q space. This signal is defined as the difference of multi-pulse signals $S(q, \tau)$ in 244 presence or absence of pump-probe time delay dispersion. Time-delay 245 dispersion is supposed to be of the order of $1000 \mathrm{fs}$. The contraction is no 246 longer perceptible at this level of jitter. 